\title{
The use of approximate methods of seismic assessment of structures
}

\author{
Maria Basdeki $^{1, *}$, Argyro Drakakaki ${ }^{2}$, and Charis Apostolopoulos ${ }^{3}$ \\ ${ }^{1}$ Civil Engineer, $\mathrm{PhD}$ candidate, Department of Mechanical Engineering \& Aeronautics, University of \\ Patras, Greece \\ ${ }^{2}$ Mechanical Engineer, PhD candidate, Department of Mechanical Engineering \& Aeronautics, \\ University of Patras, Greece \\ ${ }^{3}$ Associate Professor, Department of Mechanical Engineering \& Aeronautics, University of Patras, \\ Greece
}

\begin{abstract}
Greece is an earthquake prone area, which is also exposed to coastal environment. Most existing buildings present common characteristics, concerning quality of the materials and environmental conditions [1].The vulnerability of these structures is exteriorized under powerful seismic loads. This is because they were designed, according to older regulations, primarily to bear vertical loads and secondarily to bear horizontal loads, an indicative sign of the absence of anti-seismic design. Designing and evaluation of the seismic performance of existing structures is a really complex issue, because structural degradation phenomenon is related to both corrosion damage of steel reinforcement on RC structures and high vulnerability of masonry. Precisely, the inadequate seismic performance of masonry structures, which is recorded under intense earthquakes, is attributed to the characteristics of masonry and to the ageing phenomena of the materials. For the seismic inspection of masonry structures, both EC2 and OASP can be used [3], although there is often a great misunderstanding concerning the range of the maximum permissible interventions, the financial inability and modern perceptions of redesigning [2]. On the other hand, in the case of RC structures, there is no prediction -concerning the corrosion factor- included in the international regulations and standards. In the current study is presented an experimental procedure, concerning a RC column before and after corrosion. An estimation concerning the drop of its mechanical performance has taken place, indicating the importance of the corrosion factor. Additionally, an existing monumental masonry tower building, was subjected to seismic evaluation [4]. Both OASP and EC2 inspection methods were used. The results pointed out that, for medium-intensity earthquakes, both analytical and approximate methods are respectable and reliable.
\end{abstract}

*Corresponding author: mbasdeki@upnet.gr 


\section{Introduction}

Safety of structures is generally related to the expected service life, according to established standards and methods. However, there are plenty of factors that may be responsible for the loss of structural integrity and the reduction of residual life of a structure. It is widely known that both harsh coastal environment, which is rich in chlorides, seismicity and vulnerability of the materials used are likely to have detrimental results on the mechanical performance of the various structures. Consequences of corrosion damage on reinforced structures, and insufficient seismic response of structures that face durability issues, are widely known and analytically described in existing literature [1-3]. However, the Scientific Community's response to the issue was belated and was triggered by ageing phenomena of existing structures, corrosion damage recorded on steel reinforcement and the subsequent deterioration of the seismic bearing capacity of both steel reinforcement and structural elements demand further evaluation of the residual mechanical sufficiency and the adoption of appropriate rehabilitation techniques.

Nevertheless, it is clear enough that the evaluation of the seismic performance of the existing structures is a really complicated issue, in both the case of reinforced concrete structures, constructed prior to the recent seismic design codes [4] as well as in the case of structures made of masonry, constructed with the total absence of static study. Complicity is owed to the fact that environmental factors, which are responsible for the occurrence of corrosion damage, as well as the corrosion factor itself are not included and predicted by the existing regulations (Eurocode 2- which is used in the whole Europe- and KAN.EPEwhich is used in Greece-). Consequently, the "tools" required for the rehabilitation (preseismically or postseismically) and the prediction of the residual strength of RC structures via constitutive laws, are not available to engineers. Nonetheless, lack of encoded analysis of corrosion damage, in the existing regulations, is still justified.

However, in the present study an effort was made to attribute the importance of the corrosion factor on the performance of the various structures, either made of reinforced concrete or masonry. For this reason an experimental procedure, concerning a reinforced concrete column before and after corrosion took place. An estimation concerning the drop of its mechanical performance was demonstrated, indicating the importance of the corrosion factor. Additionally, an existing monumental tower building, made of masonry, was subjected to seismic evaluation [5]. Both OASP and EC2 inspection methods were used. The results pointed out that, for medium -intensity earthquakes, both analytical and approximate methods are respectable and reliable.

\section{Structures made of reinforced concrete}

For the goals of the present study two reinforced concrete columns were constructed and their performance under seismic loads was investigated before and after a predetermined corrosion level. In particular, repetitive loads with constantly increasing horizontal displacement were applied to the upper free end of the columns, under the simultaneous imposition of a constant axial load of $350 \mathrm{kN}$. The two columns were identically constructed with cross section equal to $300 * 300 \mathrm{~mm}$ and height equal to $1.60 \mathrm{~m}$. The baseplate was $60 \mathrm{~cm}$ tall, $120 \mathrm{~cm}$ long and $80 \mathrm{~cm}$ wide. For each column, concrete category C20/25 and dual phase B500c class were used. In each specimen were used 4 steel bars of $\Phi 16$ diameter, as main reinforcement and stirrups $\Phi 8 / 250 \mathrm{~mm}$. The concrete cover thickness was equal to $25 \mathrm{~mm}$. 
To evaluate the mechanical strength of the column, before and after corrosion, the base of one column was subjected to artificial accelerated electrochemical corrosion, in a solution consisted of $5 \% \mathrm{NaCl}$, at the height of $60 \mathrm{~cm}$ from the base. In order to achieve the desired mass loss level, each embedded rebar was corroded for 60 days, under an imposed current equal to $0.3 \mathrm{~A}$, according to Faraday's Law.

The two columns, both the reference and the corroded one, were subjected to mechanical tests, with gradually increasing displacements (drifts). Each drift was repeated three times under the simultaneous imposition of a constant axial load, equal to $350 \mathrm{kN}$. The range and the drift sequence were as follows: $0.2 \%, 0.8 \%, 1 \%, 1.25 \%, 1.5 \%, 2 \%, 2.5 \%$, $3.5 \%$ and $5 \%$, as shown in Figure 1 .

The corrosion damage developed was measured as the mass loss of the reinforcement bars, taken from the base of the corroded column. The recorded values ranged from $14 \%$ to $21 \%$ and material loss can be translated into local reduction of the nominal diameter of the steel reinforcement and local intense pitting. The uneven corrosion damage of steel reinforcement is associated with an equivalent local damage on the concrete cover, which ultimately determines the weakest area of each structural element (for example of a column) [6-7]. Seismic performance of a R.C. column is closely related to the uneven corrosion damage recorded on its reinforcement items.
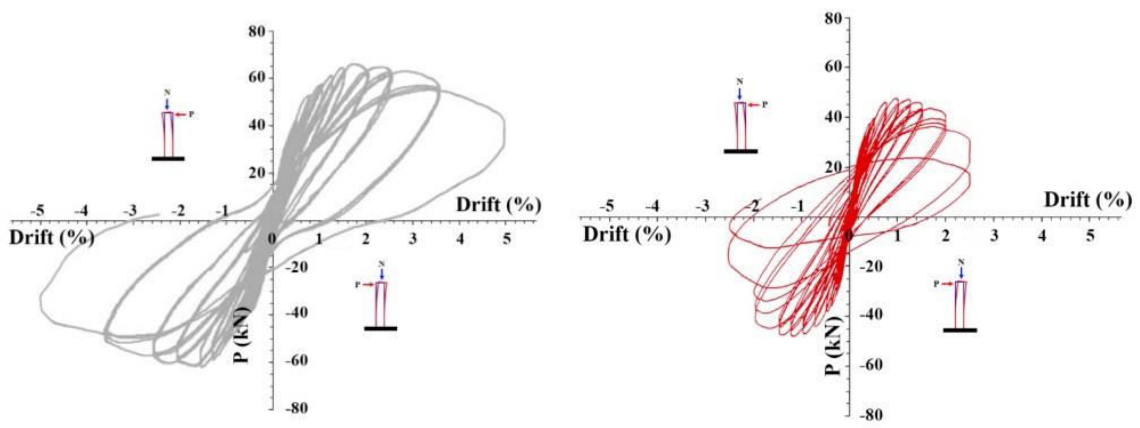

Fig. 1. Response of the reference column (left) and corroded column (right).

Analysing the mechanical response of both columns it seems that it was almost linear, up to $1 \%$ drift, although the first micro cracks were formed near stirrups. Mechanical performance of the corroded column was significantly reduced in comparison with the noncorroded one, in terms of deformation and maximum loads. The sequel differentiation concerning the maximum withdrawal capacity of both columns is credited to the corrosion rate of the steel reinforcement. At this phase, each material is expected to resist individually, withstanding both tensile and compressive loads. This fact enhances degradation phenomena, resulting in premature failure of the column not only due to concrete spalling, but also due to the buckling taking place on steel.

Additionally, although standards do not require the evaluation of the energy density $U_{d}$ of the reinforcing steel, however, the property of the energy density characterizes the damage tolerance potential of a material and may be used to evaluate the material fracture under both, static and fatigue loading conditions. For this reason, in Table 1 are listed the 
ratios of elastic and energy to the total energy for each drift (\%), as well as the corresponding horizontal displacements $\delta$.

Table 1. The ratios of elastic energies to the total energy and the $\delta$ calculations attributing to each drift $\%$ for the non corroded column (left) and corroded one (right)

\begin{tabular}{|c|c|c|}
\hline \multicolumn{3}{|c|}{ Non corroded } \\
\hline $\begin{array}{c}\text { Drift } \\
(\mathbf{\%})\end{array}$ & $\begin{array}{c}\boldsymbol{\delta} \\
(\mathbf{c m})\end{array}$ & $\begin{array}{c}\text { N1 (Elastic En./Total } \\
\text { En.) }\end{array}$ \\
\hline 1 & 1.6 & 0.96 \\
\hline 1.5 & 2.4 & 0.96 \\
\hline 2 & 3.2 & 0.96 \\
\hline 2.5 & 4.0 & 0.89 \\
\hline 3.5 & 5.6 & 0.91 \\
\hline 5 & 8.0 & 0.51 \\
\hline
\end{tabular}

\begin{tabular}{|c|c|c|}
\hline \multicolumn{3}{|c|}{ Corroded } \\
\hline $\begin{array}{c}\text { Drift } \\
(\%)\end{array}$ & $\begin{array}{c}\boldsymbol{\delta} \\
(\mathbf{c m})\end{array}$ & $\begin{array}{c}\text { N1 (Elastic En./Total } \\
\text { En.) }\end{array}$ \\
\hline 0.75 & 1.20 & 0.94 \\
\hline 1 & 1.6 & 0.94 \\
\hline 1.25 & 2.0 & 0.96 \\
\hline 1.5 & 2.4 & 0.94 \\
\hline 2 & 3.2 & 0.925 \\
\hline 2.5 & 4.0 & 0.796 \\
\hline
\end{tabular}

From Table 1 it becomes clear that the ratio Elastic Energy/Total Energy of the corroded column is lower than the ratio of the non-corroded column at the same drift. This is because, higher embrittlement owed to corrosion factor, diminishes the elasticity of the structure. Finally, the reduction of the energy reserves, due to the upcoming embrittlement, results in premature failure of the reinforced concrete column. In any case, the full analysis of the performance of the two reinforced concrete structures has already been presented in the $18^{\text {th }}$ Concrete Conference, which took place in Athens [8]

Nevertheless, according to the previously demonstrated results, it becomes clear that great drop can be recorded on the performance of such structures, which are exposed to both aggressive environment and to seismic activity. Consequently, there is a great need to enrich existing regulations and standards with the prediction of corrosion damage, concluding in more reliable intervention and rehabilitation.

\section{Structures made of masonry}

Masonry represents one of the most ancient and longest surviving structural composite material, yet knowledge about its structural integrity and mechanical behaviour is quite limited. This contradiction can be explained by the fact that masonry is a diverse composite material, thus fundamental research of individual component substances - brick and mortar - is required as to understand their mechanical interaction as part of the structure. A strong deterrent to setting an international regulatory framework for further analysis of physical and mechanical properties of masonry is due to its diversity and therefore there is great difficulty in standardisation of materials and test methods. Experience gained from recent earthquakes indicates that structures made of masonry present satisfactory strength to ageing. Thus, their seismic response is conditioned by numerous factors, including symmetry of the structure, the thickness of masonry, the existence or absence of horizontal cantrail and the number and arrangement of openings.

The correlation of existing structures made of masonry necessarily differentiate from new buildings owing to several reasons :

- materials and types of masonry do not meet the requirements of Eurocode 6,

- the vast majority of these structures were built with the absence of static study bringing a defective design in their bearing structure, many of them are often difficult to detect and investigate. 
Therefore, besides Eurocode 8, a parallel regulatory framework needs to be developed with appropriate adjustments for the assessment and redesign of existing structures made of masonry, such as approximate method of OASP. Organisation of Earthquake Planning and Protection (OASP) is a legal entity of public law, whose purpose is the elaboration and design of greek antiseismic policy within the framework of the governmental guidelines. OASP has already developed a 3-level practice for the classified seismic assessment of buildings:

1. a rapid visual preseismic inspection (first degree inspection)

2. a secondary preseismic inspection and

3. a tertiary preseismic inspection.

The rapid visual inspection constitutes a simplified method applied to vast amount of buildings and therefore must be regarded as of limited reliability. Hence, the scope of secondary preseismic inspection form those buildings which obtained rating below a threshold level.

\subsection{Analysis in terms of displacement}

It is a standard practice for assessment of existing structures made of masonry a finite element analysis to be carried out, in order to determine the elastic displacements of the structure. As it is known, the actual (post-elastic) displacements derive from those calculated through the analysis, multiplied with behaviour factor q. In order to evaluate the inelastic displacements of the structures, a simplified energy equivalence is used via elastic and inelastic analysis, as shown in Figure 2. [9]

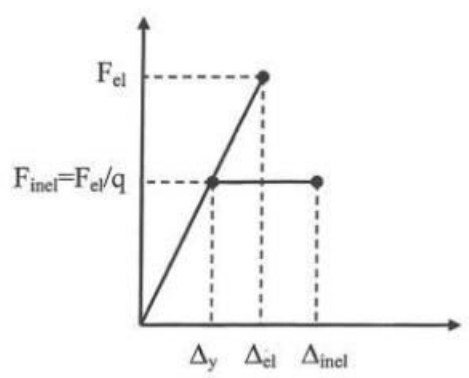

Fig. 2. Diagram of force - displacement for elastic and inelastic analysis.

$$
E_{\mathrm{el}}=E_{\text {inel }} \rightarrow \frac{1}{2} \Delta_{\mathrm{el}} F_{\mathrm{el}}=\frac{1}{2}\left(\Delta_{\text {inel }}+\Delta_{\text {inel }}-\Delta_{\mathrm{y}}\right) \frac{F \mathrm{el}}{q} \rightarrow \Delta_{\mathrm{el}} q=2 \Delta_{\text {inel }^{-}} \Delta_{\mathrm{y}}
$$

The following equations about the correspondence between elastic and inelastic displacements originate from the equation above, due to energy equivalence with behaviour factor q equal to 1.5 , which is a representative value for masonry structures. More specific,

$$
\begin{aligned}
& \Delta_{\mathrm{y}} / \Delta_{\mathrm{el}}=\left(F_{\mathrm{el}} / q\right) / F_{\mathrm{el}} \Delta_{\mathrm{y}} \rightarrow \Delta_{\mathrm{y}}=\Delta_{\mathrm{el}} / q= \\
& \Delta_{\mathrm{el}} q=2 \Delta_{\text {inel }^{-}} \Delta_{\mathrm{y}} \rightarrow \Delta_{\text {inel }}=\left[\left(q^{2}+1\right) / 2 q\right] \Delta_{\mathrm{el}}
\end{aligned}
$$

\subsection{Secondary pre seismic audit}

As mentioned above, the objective of the secondary preseismic audit is an hierarchical reclassification of masonry structures, based on a complete surveying and evaluation of 
technical characteristics while duly taking into account "social" criteria, based on occupancy, market value and possible historical/architectural value of the buildings. This inspection is going into more details and requires full accessibility of the building, drawing up drafts of geometry and building pathology, optical evaluation and basic calculations for the quantitative assessment of significant indexes, with the absence of simulation procedures.

The approximate method of OASP [10], with all necessary amendments or additions, is in line with the research of Tassios T. and Vintzileou E. [11], a pseudo-quantitative method in order to assess the relative seismic risk of historical masonry buildings. This method is more elaborated than a rapid visual screening, where the classification of the building is based on the comparison of a broader action effect $(\mathrm{H})$ and the corresponding resistance $(\mathrm{R})$ in accordance with the fundamental inequality of safety considered :

$$
H \ngtr R \text { or } H / R-1 \ngtr 0
$$

The abovementioned quantity $\mathrm{H} / \mathrm{R}-1$ is considered to be the basic inadequacy index of the building, which in the vast majority of existing structures is expected to have positive values due to high seismic vulnerability, especially in the light of current scientific and technical knowledge under powerful seismic loads. This index is an estimator of vulnerability of the structure, whereas $\mathrm{H}$ and $\mathrm{R}$ will be conventionally estimated by means of empirical coefficients. Yet it cannot be used as the seismic capacity coefficient of the structure, comparing to the one resulting from thresholds set by Eurocode 8. The estimation of seismic loading $\mathrm{H}$ will be calculated on the basis of data included in the Greek Seismic Code, confining zoning and local soil conditions. The estimation of the resistance $\mathrm{R}$ will be calculated taking into account the following characteristics, including shear resistance of masonry walls within their plane, normalised length of openings of the walls along the same direction, existence of confinement, previous light damages, connection between perpendicular walls, out-of-plane resistance of main walls and available diaphragms.
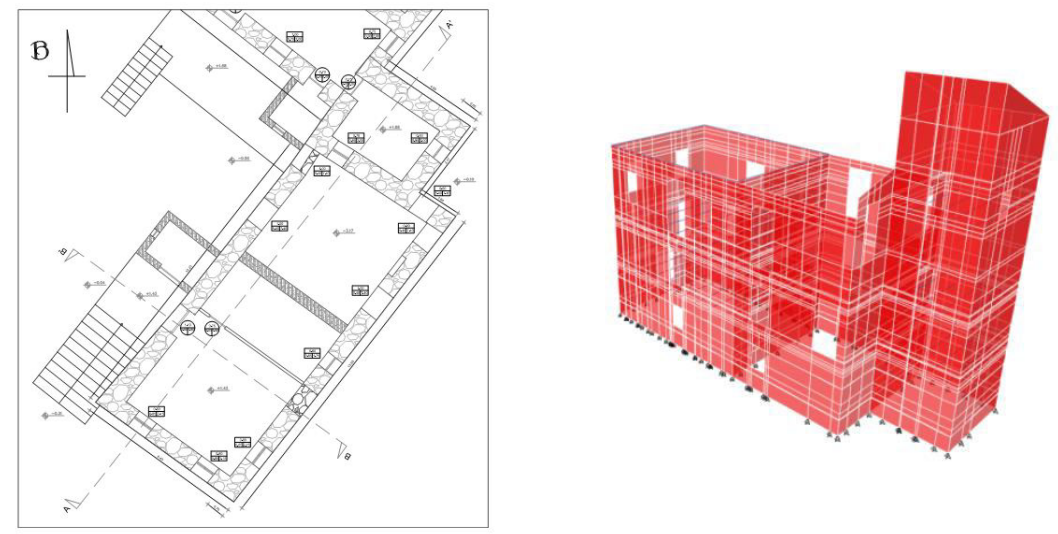

Fig. 3.General plan of the building (left) - model of finite element analysis (right)

A three-storeyed masonry building, constructed approximately in 1750 at a mountainous village of Achaia Prefecture, Greece [5], has been used for the analytical work of the present study. Figure 3 depicts the plan of the building model, with dimensions 
$13.65 \mathrm{~m} \times 6.45 \mathrm{~m}$. Further details concerning the construction of the building model and the mechanical characteristics of the materials can be found in dissertation thesis [5].

The calculated inadequacy index for this building, according to OASP, was equal to 1.67 contrary to one according to Eurocode 8, which was equal to 1.28. Moreover, Figure 4 depicts diagrammatically the inelastic out -of -plane displacements calculated according to EC8 in the façade of the building (left) and the actual situation of it (right).
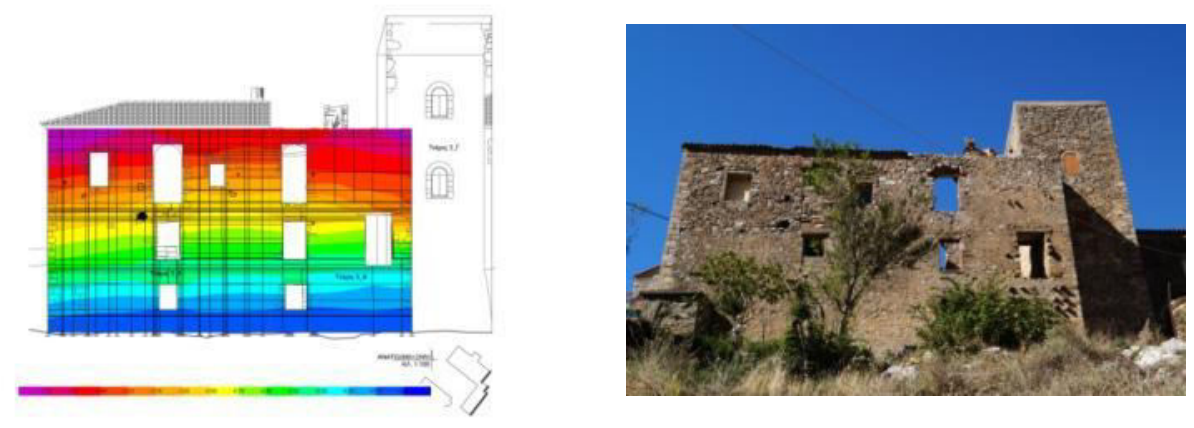

Fig. 4.Inelastic analysis (left) - Actual situation (right)

\section{Conclusions}

The present manuscript demonstrates the importance of approximate methods of assessment of both RC structures and structures made of masonry. Analytically, the mechanical performance of a reinforced concrete column before and after corrosion has been evaluated, under the influence of increasing horizontal displacement and the simultaneous imposition of a constant axial load. Additionally an approximate method for the assessment of the seismic capacity of existing buildings has taken place. The approximate method of OASP [10] has been followed in order to assess a masonry building and a comparison has been made with factual situation of it and finite element analysis [5]. The conclusions of both aspects of the present study are as follows:

- Corrosion of steel is a highly deteriorating and aggressive factor for concrete structures, since the drop in mechanical properties affects negatively the seismic loading capacity and the structural integrity of RC members.

- The uneven corrosion damage determines the critical point of the reinforced concrete structures. At the same time it greatly affects their seismic performance as well.

- Reinforced concrete sections are unable to cope with the high deformation demands, owed to loading, resulting not only in the degradation of loops, but also in severe degradation of each individual deformation; namely, the elastic and the plastic deformation.

- The approximate method of OASP [10] predictions of storey drift displacements for medium earthquakes appear to be more consistent with actual situation in the aftermath of the earthquake. More specific, the results showed that the damages recorded due to seismic action were found to be in satisfactory compliance with the proposed by OASP method, as opposed to the results based on EC2, where the damages were found clearly minor. 
- $\quad$ The method of OASP does not take into account the real mechanical properties of the masonry, due to lack of experimental testings. Therefore, Young's modulus of elasticity has a significant impact on the method, while possible reduction of it leads to significant increase of the inadequacy index of the structure according to EC8. Thus, inadequacy index according to EC8 are conservative in compare to those according to OASP.

- Greater convergence of the two methods can be seen for values larger than 1.5 of the behaviour factor.

\section{References}

1. Apostolopoulos, C.A., Demis S., Papadakis, V.G., Constr. Build. Mater., Vol.38, pp.139-46 (2013)

2. Papadopoulos, M.D., Apostolopoulos, C.A., Zervaki, A.D., Haidemenopoulos, G.N., Jour. Constr. Build. Mater., 25, pp 3367-3374 (2011).

3. Almusallam A., Constr. Build. Mater., Vol. 15, No.8, pp. 361-368 (2001)

4. Afsar E., Kashani M.M., Struct. Infrastruct. E., Vol.14, No 6, pp.714-729 (2017)

5. Basdeki M., Seismic assessment of an existing monumental building made of masonry according to EC8 and method of OASP. Dissertation Thesis, Patras (2014) [In Greek]

6. Rinaldi Z., Valente C. Pardi L., Struct. Infrastruct. E., Vol. 4, No.2, pp.139-152 (2008)

7. Bertolini L., Struct. Infrastruct. E., Vol. 4, No.2, pp. 123-137 (2008)

8. Basdeki, M., Drakakaki, A., Apostolopoulos, C., $18^{\circ}$ Concrete Conference, Athens (2018) [In Greek]

9. Apostolidi, E., Dritsos, S., Tsirlis, F. and Armenis, G., Technical Chamber of Greece (TEE) - Regional Department of Corfu (2013) [In Greek]

10. O.A.S.P. (Dritsos, S., Ignatakis, Ch., Panagiotopoulou, D., Spiliopoulos, A.) Secondary preseismic inspection of structures made of masonry, Athens (2012)

11. Tassios, T., Vintzileou, E., ETEPAM, 2th Panhellenic Restoration Conference, Athens (2009) [In Greek] 